\title{
Methodology for estimating building integrated photovoltaics electricity production under shadowing conditions and case study
}

\author{
Daniel Masa-Bote , Estefanía Caamaño-Martín
}

\begin{abstract}
A B S T R A C T
Building integrated photovoltaic (BIPV) systems are a relevant application of photovoltaics. In countries belonging to the International Energy Agency countries, 24\% of total installed PV power corresponds to BIPV systems. Electricity losses caused by shadows over the PV generator have a significant impact on the performance of BIPV systems, being the major source of electricity losses. This paper presents a methodology to estimate electricity produced by BIPV systems which incorporates a model for shading losses. The proposed methodology has been validated on a one year study with real data from two similar PV systems placed on the south façade of a building belonging to the Technical University of Madrid. This study has covered all weather conditions: clear, partially overcast and fully overcast sky. Results of this study are shown at different time scales, resulting that the errors committed by the best performing model are below $1 \%$ and $3 \%$ in annual and daily electricity estimation. The use of models which account for the reduced performance at low irradiance levels also improves the estimation of generated electricity.
\end{abstract}

\section{Contents}

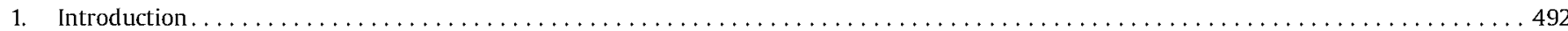

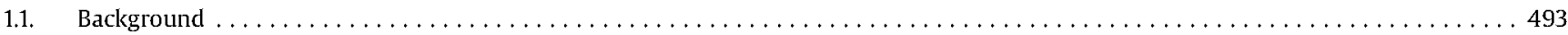

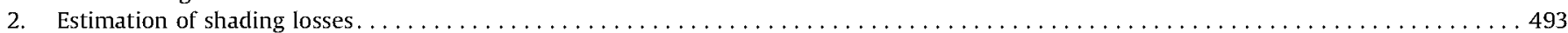

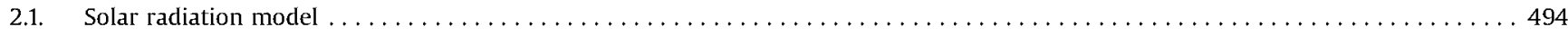

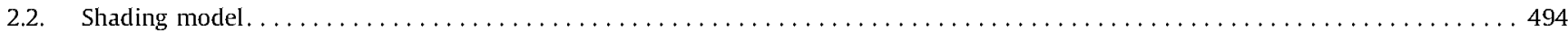

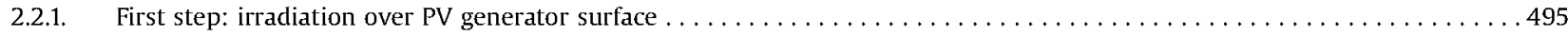

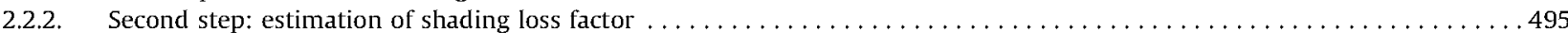

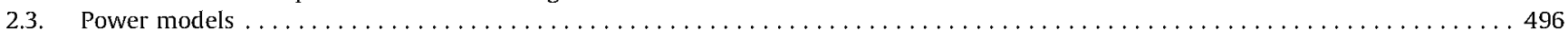

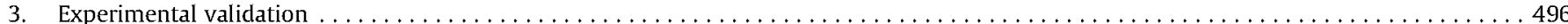

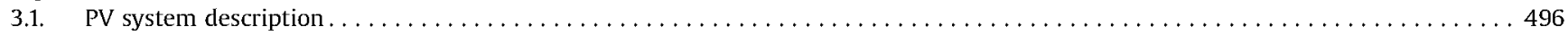

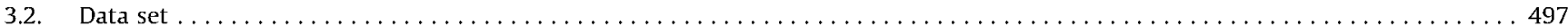

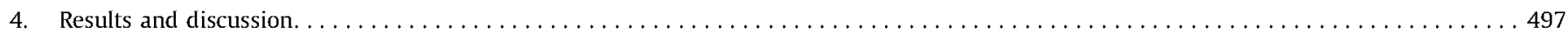

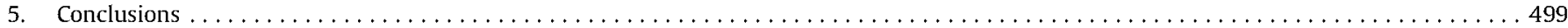

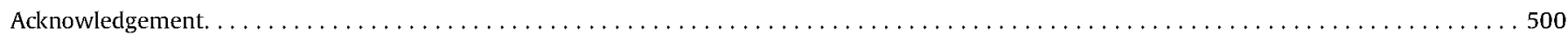

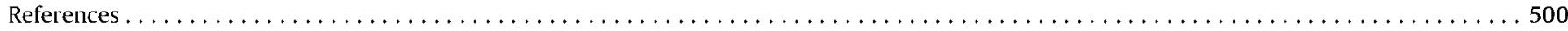

\section{Introduction}

Over the past two decades the number of grid connected PV systems has experienced an important growth. According to the
International Energy Agency IEA [1], between 1992 and 2010 the average growing rate of installed $\mathrm{PV}$ power was $39 \%$ in its member countries. This growth has been due to the inherent benefits of renewable energies and supporting polices by public entities, mostly based on feed-in-tariffs. The total cumulative power installed in IEA countries at the end of 2010 was $35 \mathrm{GW}$. Of this capacity, nearly $24 \%$ was integrated in or associated to buildings.

Despite progressive reductions of feed-in-tariffs in most countries, it is expected for BIPV systems to keep their growth rate. 
With rising prices of retail electricity and decreasing costs of PV, grid parity with commercial electricity will soon become a reality, especially in urban residential areas. This fact, together with new policies that enhance self-consumption (currently under discussion in Spain [2]) points that building integrated photovoltaic (BIPV) systems will become more relevant in the future. Also to be mentioned is the new European Energy Performance of Buildings Directive [3], where PV can definitely play a new role in the socalled "nearly zero-energy buildings" which are described in the directive as buildings with a "very high energy performance. [...] The nearly zero or very low amount of energy required should be covered to a very significant extent by energy from renewable sources, including energy from renewable sources produced on-site or nearby".

The increase in installed PV power demands for simple, accurate models able to predict the electricity expected from a system. These models should be confronted with actual data from existing installations to validate them. However, in the literature there is a lack of specific studies about performance of PV systems integrated in buildings [4-6]. As a consequence there is also a lack of models which describe system behaviour with reasonable accuracy.

Location determines the performance of a PV system in two ways: climate and obstacles surrounding the system. Available irradiance and ambient temperature are set by climate while obstacles may reduce the effective irradiance over the PV generator. Orientation and slope of the PV generator surface also affect the effective irradiance received. However, while energy loss due to small deviations on orientation and slope from optimal position have low sensitivities [7], the impact of shading on system performance is relevant.

Shadows over BIPV systems are casted by nearby objects such as trees, utility poles, surrounding buildings or even the building itself on which the system is integrated. Regardless their nature, these obstacles are characterized by irregular shape and closeness to the PV generator. The irregular shape makes its modelling and incorporation into the obstacle profile a complex and tedious task. The closeness of obstacles to the PV generator means that an important fraction of the sky dome is occupied by these obstacles and shading has a high impact on BIPV systems. Therefore, a practical estimation method of BIPV systems performance should include an accurate methodology for evaluating shading losses. This paper presents a methodology to estimate the electricity produced by a BIPV system based on the combination of a novel model for the estimation of shading losses with well-known validated models for estimation of electricity production. The performance of this methodology has been assessed with a case study: The hourly electricity produced over one year by two BIPV systems integrated in the same building, one heavily shaded while the other is relatively free of shadows, has been estimated using the methodology and compared to the actual, measured values.

The structure of the remainder of the paper is as follows. Section 2 describes the procedure to estimate system output, paying special attention to the model for shading losses. Section 3 describes a BIPV system used to validate this methodology. Results and comments on this validation are given in Section 4. Finally, conclusions on the results and methodology usage are given in Section 5.

\subsection{Background}

Any method for the estimation of shading losses is based on the combination of two steps that can be considered as two independent processes:

- First, the irradiance over the surface of interest - the PV generator - is obtained. This step needs as input two sets of data: a series of annual irradiation, typically hourly values, and a description of the obstacles surrounding the PV generator. The comparison of obstacle profile and sunpath determines at which hours the generator is obscured by shadows.

- On the second step the electricity that can be extracted from the PV generator is estimated. This is expressed in terms of a dimensionless factor called shading loss factor: $L_{S H}$ which can be added to the various losses also existing in a PV system.

On the past decades several methods developed to address shading losses have been published on the literature. Some of these methods include the two steps described above or are limited to only one of the steps.

Alonso-García [8,9], Di Pizza [10], Karatepe [11], Kawamura [12] and Rauschenbauch [13] provide theoretical models to simulate the behaviour of PV modules and generators in conditions of nonuniform radiation. However, they do not provide methods to obtain the distribution of shadows over the surface under study. The majority of these models, the only exception being Kawamura's one, take into account the effect of bypass diodes. Also, three authors (Rauschenbauch, Kawamura and AlonsoGarcia) include the effect of the inverse $I-V$ characteristic of the shaded cell to estimate the $I-V$ overall curve of the module or generated affected by shadows.

Other authors, on the contrary, provide models to estimate the effective irradiation, that is, after incorporating shading effects but these models do not estimate the electricity losses in which the PV system incurs due the effect fn inhomogeneous irradiation. Drif [14] proposes a model based on geometrical description of obstacles in which considers each component of solar radiation, beam and diffuse, separately. Drif's model has been validated experimentally on a PV system located at Jaen University (Spain). Niewenda [15] introduces a software tool, SOMBRERO that allows to obtain the irradiation over any surface in the presence of obstacles. This tool is not limited exclusively to PV systems and can be combined with other tools (SUNCODE, TRNSYS) to evaluate the impact of shadows on passive applications of solar energy.

Finally, Woyte [16] and Oozeki [17] analyze shading losses from the perspective of electricity production of the overall PV system, focusing their analysis on electricity losses rather than irradiation losses. Woyte studies the impact of shadows on a PV generator according to its electrical configuration: generator formed by several strings connected in parallel to one central inverter, each string connected to an inverter or AC modules. For large obstacles whose shadow covers a significant section of the generator Woyte does not find differences in performance between the three configurations. Oozeki introduces a model to estimate electric losses caused by shadows, as well as other losses, based on the monitoring of the system during one year. Oozeki's model great advantage is that it does not require a characterization of the obstacles. On the other hand, its main drawback is that it requires a time span of one year and it cannot be used to estimated shading losses during the design phase of a PV system.

\section{Estimation of shading losses}

Fig. 1 shows an outline of the procedure proposed to estimate PV system output. Shading losses are the most relevant factor on BIPV systems performance and, therefore, the model to estimate them is the core of the methodology shown in the figure. The model for the estimation of shading losses has been developed according to the following objectives:

- Accuracy: obstacle profile, despite its complexity, must be characterized accurately by the methodology; this will result in better estimation of associated losses. 


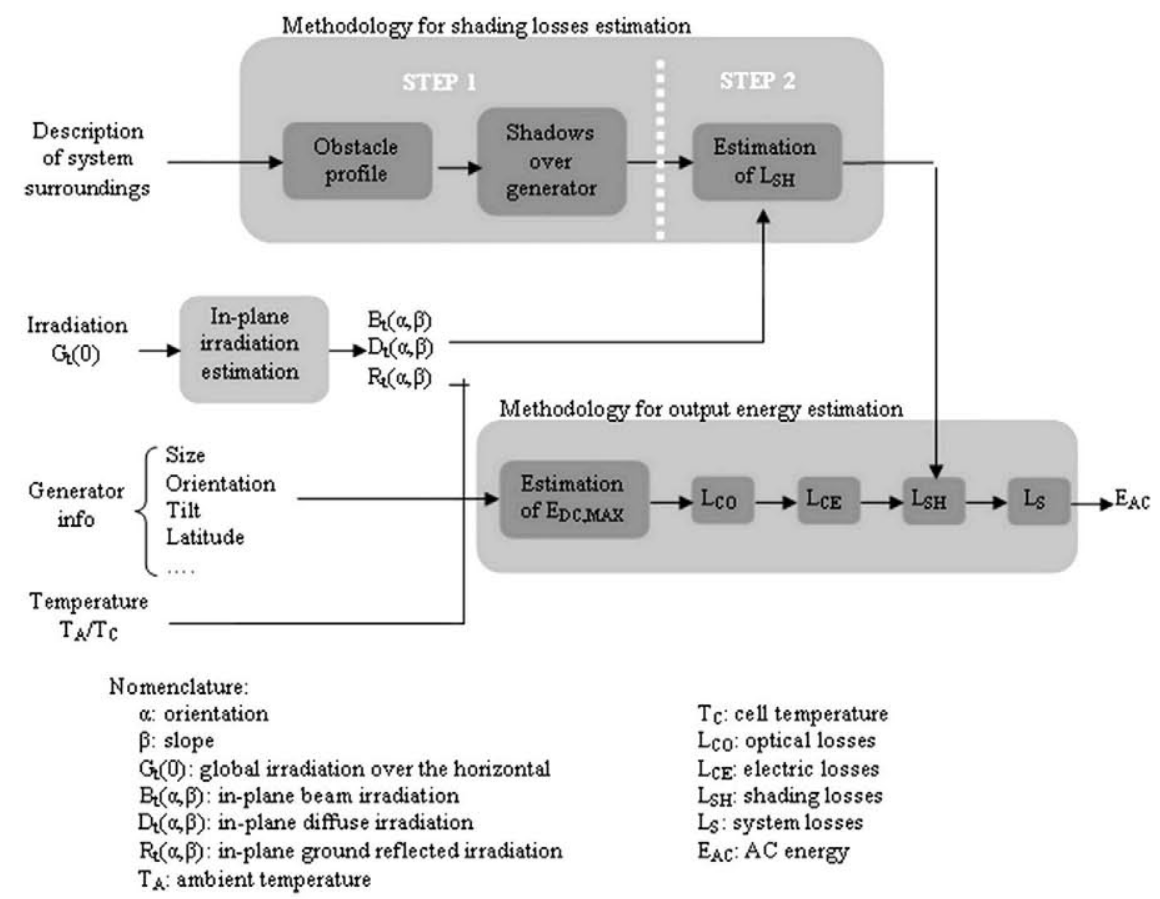

Fig. 1. Flowchart of methodology for system output estimation.

- Simplicity: the methodology must be flexible and simple enough to describe obstacles, however complex they are, using few and easy-to-understand information.

The models in Fig. 1 are described in detail below.

\subsection{Solar radiation model}

The three components of solar irradiation over an arbitrarily oriented and tilted surface can be estimated if the global irradiation over the horizontal plane is known. For this purpose scheme proposed by Liu and Jordan [18] is used: beam and diffuse components of horizontal irradiation are estimated from global irradiation through the existing correlation between clearness index and diffuse fraction. The use of a local correlation, when available, is preferred over the use of a global correlation $[7,19]$.

Beam and albedo irradiation components over an inclined surface, $B_{t}(\alpha, \beta)$ and $R_{t}(\alpha, \beta)$ respectively, during a given time period $t$ are estimated in a straightforward manner as shown in Eqs. (1) and (2), where $B_{t}(0)$ is beam component of horizontal irradiation, $G_{t}(0)$ is global component of horizontal irradiation, $\theta_{S}$ is incidence angle, $\theta_{z s}$ is solar zenith angle, $\alpha$ is orientation angle ( $0^{\circ}$ for south orientation and positive to the west), $\beta$ is tilt angle ( $0^{\circ}$ for horizontal surface) and $\rho$ is surface albedo:

$B_{t}(\alpha, \beta)=B_{t}(0) \cdot \max \left(0, \cos \theta_{S}\right) / \cos \theta_{z s}$

$R_{t}(\alpha, \beta)=G_{t}(0) \cdot(1-\cos \beta) \cdot \rho / 2$

Perez model is used to estimate diffuse irradiation [19]. This model has been selected over similar models due to its better performance [7,20-22].

The model described so far needs global irradiation over a horizontal surface to estimate the components of irradiation over a tilted surface which is, in turn, necessary to estimate shading losses. However, in BIPV systems, if irradiation is measured it is always available on the same orientation and tilt as the PV generator. Therefore, a procedure to estimate horizontal irradiation from in-plane irradiation is necessary since there are no models in the literature for the correlation between diffuse and global components of irradiation over tilted surfaces. A novel approach to obtain values of horizontal irradiation based on selecting days from a typical meteorological year (TMY) from the PV system location is proposed. This approach has the advantage that it relies on data sets that characterize statistically the local climate conditions and are increasingly available [23,24]. Every day of measured tilted irradiation is replaced by a day from the TMY. It is not possible to replace an actual day with the same day same date - from the TMY because the diffuse fraction of both days will not be the same. Instead, every day is replaced by the day from the TMY which is most similar to it. Similarity between days is estimated by the function in Eq. (3), $f_{S}$. The first term inside the brackets, $\rho_{\text {TMYDATA }}$, is the correlation coefficient between both days; this term assures that the days from the TMY will have a similar energy distribution than the days from the recorded set. While correlation coefficient provides days with similar power profiles, it does not guarantee that these days have the same irradiation. For this purpose a comparison between daily energies is included as second term; $G_{d, T M Y}(0)$ is daily irradiation of the day from the TMY, $G_{d, \text { data }}(\alpha, \beta)$ is daily irradiation of the actual day and II II represents absolute value function. The search inside the TMY is restricted to a window with a size of $2 w+1$ days, centred on the day to be replaced and extended $w$ days ahead and behind of it. From within this interval the day that maximizes $f_{S}$ is selected:

$f_{S}=\left\{\rho_{T M Y, D A T A}+\left[1-\left\|\left(G_{d, T M Y}(0)-G_{d, \text { data }}(\alpha, \beta)\right) / G_{d, \text { data }}(\alpha, \beta)\right\|\right]\right\} / 2$

\subsection{Shading model}

The proposed methodology for shading losses estimation is based on the following concepts:

- Irradiation components are considered separately. Global irradiation is separated into its three components: beam, diffuse and ground-reflected.

- Obstacles and PV generator are modelled as three dimensional objects. On BIPV systems the size and distance of the obstacle to the generator are frequently similar to the dimensions of the generator itself. Therefore, it cannot be assumed that the 


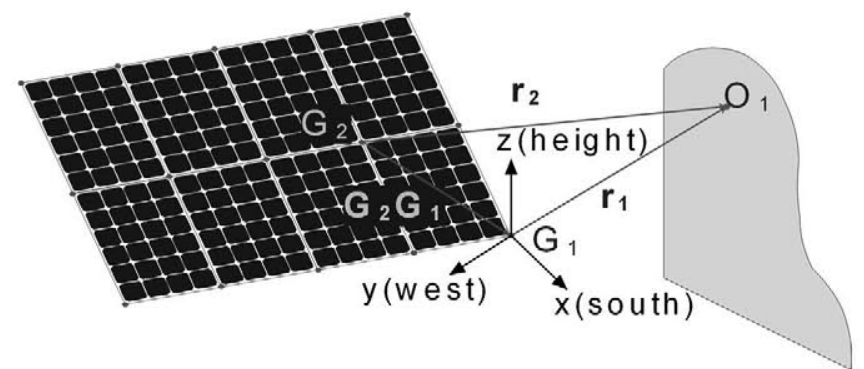

Fig. 2. Grid defined over the PV generator surface for shading losses estimation and translation procedure of obstacle coordinates between points inside the PV generator.

generator is constrained to a single point; instead, the generator is considered as a 2 dimensional surface. In addition, the PV generator will not be evenly lighted when shaded: some parts will be covered by shadows while others will be illuminated directly by the Sun. In contrast, the much simpler assumption that the generator is dimensionless only allows for two possible states: fully illuminated or fully covered by shadows.

- Shading losses are estimated using geometrical considerations. Although the effect of non-uniform lighting conditions on the electrical behaviour of a PV system is not linear with the surface fraction covered by shadows [8-13] the model developed only takes into account the geometrical configuration of the PV generator, not its electrical configuration. The use of an electrical model to account for shading losses in a PV system requires, in addition to estimate parameters associated to the model, that physical and electrical configuration of both PV modules (number of cells, series-parallel configuration, size and number cells protected by a single bypass diode) and generator (series-parallel configuration, placement of modules along their long or short side). This situation can be complicated even further if PV modules are placed following an irregular pattern, as it is the case on some BIPV systems for constructive or aesthetic purposes. On the contrary, the geometric approach proposed provides a less complex shading model: the only information needed concerning the PV generator is its physical dimensions, orientation angle $(\alpha)$ and tilt angle $(\beta)$.

As shown in Fig. 1, shading losses are estimated following a two-step process. Both steps are described below.

\subsubsection{First step: irradiation over PV generator surface}

First step goal is to estimate the incident irradiation on any point of the PV generator surface. This step starts by defining a grid of points over the PV generator. Obstacles and generator sizes are assumed to be of similar magnitude, meaning that every point of the generator "sees" a different obstacle profile. The points in the grid are selected so that they are coincident with the corners of the modules in the generator (as indicated by the grey dots in Fig. 2). For every point in the grid an associated obstacle profile is calculated. An obstacle profile is a two dimensional function that assigns to every point in the sky a binary value: 0 if that point is occupied by an obstacle, 1 otherwise. The range of this function comprises values of azimuth from $-180^{\circ}$ to $180^{\circ}$ and elevation from $0^{\circ}$ to $90^{\circ}$. The obstacle profile is defined as discrete function so it can be easily computed by software. The resolution is set at $0.5^{\circ}$ for azimuth and $0.25^{\circ}$ for elevation. The obstacle profile function is formally defined in Eq. (4), where $\psi$ is azimuth and $\gamma$ is elevation.

$O P(\psi, \gamma)=\left\{\begin{array}{ll}0, & (\psi, \gamma) \ni \text { obstacle } \\ 1 & \text { otherwise }\end{array} ; \quad \psi=[-180,180], \quad \gamma=[0,90)\right.$

Fig. 2 shows the procedure to obtain the obstacle profile for every point in the grid. Initially, the coordinates of all relevant obstacle points relative to one point of the PV generator surface, $G_{1}$ in Fig. 2, are known. To obtain the obstacle profile seen from point $G_{2}$ all points in the obstacle are translated from the coordinate system centred in $G_{1}$ to a new coordinate system whose origin is $G_{2}$. The translation vector is the coordinates of $G_{1}$ with respect to $G_{2}$; vector $\mathbf{G}_{2} \mathbf{G}_{1}$. For example, the coordinates of point $O_{1}$, which belongs to the obstacle, with respect to $G_{2}\left(\mathbf{r}_{2}\right)$ are obtained by adding the translation vector to the coordinates of $O_{1}$ relative to $G_{1}$ :

$r_{2}=r_{1}+G_{2} G_{1}$

Once all points of the obstacle have been translated, their rectangular coordinates are converted into spherical coordinates: elevation $(\gamma)$ and azimuth $(\psi)$. The repetition of this translation process for all the points in the grid provides a set of obstacle profiles. This set of obstacle profiles contains all the necessary information to know the distribution of shadows over the PV generator surface at any moment.

\subsubsection{Second step: estimation of shading loss factor}

The second step estimates losses due to shading on a PV generator from the set of obstacle profiles generated in the previous step. It starts by evaluating individually the effect of obstacles on every component of solar irradiation: beam $B$, albedo $R$, circumsolar diffuse $D_{C S}$, isotropic diffuse $D_{I S}$ and horizon band diffuse $D_{H}$. Each component has a different behaviour regarding obstacles, by evaluating them separately five partial shading loss factors are obtained.

- Beam and circumsolar diffuse irradiation share in common that they come from the Sun position in the sky. Therefore, their respective shading loss factors, $L_{\mathrm{SH}, \mathrm{B}}$ and $L_{\mathrm{SH}, \mathrm{CS}}$, are estimated in the same way. The grid superimposed to the PV generator divides its surface into rectangular regions. The behaviour of each region is governed by the four points at its vertexes: if one region has two or more of its vertexes in the shaded part of the PV generator this region does not contribute to the electricity production of the generator. The partial shading loss factor for beam and circumsolar diffuse is then defined as

$L_{S H, B}=L_{S H, C S}=\frac{\{\text { number of shaded regions }\}}{\{\text { total number of regions }\}}$

The reason why a shaded region does not affect the behaviour of non-shaded regions is because it is assumed that bypass diodes effectively isolate the effect that shaded modules have on non-shaded modules of the PV generator. By identifying every module with a region it is assumed that only one bypass diode is installed per module that limit the electrical power losses to the ratio between the area protected by one single diode and total PV module area $[25,26]$. This is a conservative approach since modules usually have several bypass diodes installed. However, with this assumption it is not necessary to know how many bypass diodes a module has, thus reducing the complexity of shading effects calculation [27].

- Isotropic diffuse irradiation comes from all points of sky hemisphere. For every point in the grid its associated loss factor $l_{S H, I S}$ is the ratio between the solid angle of the obstacles and the total solid angle of the sky hemisphere seen by the PV 
generator. The subscript in $I_{S H, I S}$ denotes that this loss factor is defined for a single point not for the whole PV generator. It must be noted that the amount of sky seen by a PV generator depends on its tilt angle. It is considered for convenience that, in the estimation of the solid angle occupied by obstacles, the radial distance is equal to 1 . Therefore, it is omitted in Eq. (7). The shading loss factor for the whole PV generator, $L_{S F, I S}$, is the average of all partial factors obtained from the PV generator points defined by the grid:

$l_{S F, I S}=\frac{\text { (solid angle of obstacles) }}{\text { (solid angle of sky hemisphere) }}=\frac{\sum \psi \Sigma \gamma\{O P(\psi, \gamma) \cdot \sin \psi \cdot \Delta \psi \cdot \Delta \gamma\}}{2(\pi-\beta)}$

$L_{S F, I S}=\operatorname{mean}\left(l_{S F, I S}\right)$

- Horizon band diffuse and albedo, although originated from different physical phenomena, have the same behaviour: they both come from the horizon line. The partial loss factors $\left(l_{S H, H}\right.$; $\left.l_{S H, R}\right)$ for every point in the grid are defined as the fraction of the horizon seen by the PV generator. A PV generator always sees half of the horizon line, an arc of $\pi$ radians centred on generator orientation, not depending on its inclination and orientation. As before, the loss factors, $L_{S F, H}$ and $L_{S F, R}$, are the averages of the corresponding partial loss factors:

$I_{S F, H}=I_{S F, R}=\frac{\text { (angle of obstacles) }}{\text { (angle of half horizon) }}=\frac{\sum_{\psi}\{O P(\psi, \gamma) \cdot \Delta \psi\}}{\pi}$; with $r=1, \gamma=0$

$L_{S F, H}=\operatorname{mean}\left(l_{S F, H}\right) ; L_{S F, R}=\operatorname{mean}\left(l_{S F, R}\right)$

Finally, the overall shading loss factor, $L_{S F}$, is obtained from the weighted combination of the loss factors estimated for every solar irradiation component:

$L_{S F}=\left(B_{t} \cdot L_{S F, B}+D_{t C S} \cdot L_{S F, C D}+D_{t I S} \cdot L_{S F, I S}+D_{t H}\right.$
$\left.\cdot L_{S F, H}+R_{t} \cdot L_{S F, R}\right) / G$

\subsection{Power models}

The electricity produced by a PV system can be determined by estimating first the maximum DC electricity that can be delivered by the PV generator and later subtracting losses related to different physical phenomena:

- Shading losses $L_{S H}$ : these losses are described in Section 3.2.

- Optical losses $L_{C O}$ : losses caused by the reflection of incident light when incidence angle is different than perpendicular. The model proposed by Martin has been used [28].

- Electrical losses $L_{C E}$ : electrical losses include losses due to voltage drops in wires on the DC side and PV modules mismatch. In this study electrical losses are included in the models for maximum DC electricity estimation and they are not considered separately.

- System losses $L_{S}$ : these losses account for the instantaneous DC-AC conversion efficiency of the inverter. The widely used model proposed by Schmidt has been selected [29] because its theoretical-practical approach, which allows to estimate model's parameters from actual measurements of inverter operation. This model is presented in the following equation:

$\eta=p_{o} / p_{i}=p_{o} /\left(p_{o}+\right.$ losses $)=p_{o} /\left(p_{o}+k_{0}+k_{1} \cdot p o+k_{2} \cdot p_{o}^{2}\right)$

where $\eta$ is inverter efficiency, $p_{o}$ is normalized inverter output power, $p_{i}$ is normalized inverter input power and $k_{i}$ are coefficients associated to conversion losses: $k_{o}$ represents inverter self consumption, $k_{1}$ represents inverter losses whose dependence on power is linear (diode consumption, switching devices) and $k_{2}$ represents losses whose dependence on power is quadratic (wires, resistors, coils, etc.).

- Thermal losses: since the effect of temperature on system yield is included in the models for maximum DC electricity estimation thermal losses are not accounted independently.

AC electricity produced by a PV system is estimated by the following equation, which incorporates the losses previously defined:

$E_{A C}=E_{D C, M A X}\left(G, T_{C}\right) \cdot\left(1-L_{S H}\right) \cdot\left(1-L_{C O}\right) \cdot\left(1-L_{C E}\right) \cdot\left(1-L_{S}\right)$

Maximum DC electricity produced by a PV generator, $E_{D C, M A X}$, depends only on operation conditions: irradiance and cell temperature. In this study two different models for the estimation of maximum DC electricity have been tested. The first model was proposed by Osterwald [30]. This model combines simplicity and accuracy and performs better than more complex models for crystalline silicon modules [31]. Osterwald model is presented in the following equation:

$E_{D C, M A X}=P_{n G} \cdot G_{t} \cdot\left[1-\gamma_{P} \cdot\left(T_{C}-T_{C}{ }^{*}\right)\right] / G^{*}$

where $G_{t}$ is irradiation, $G^{*}$ is irradiance at standard test conditions $\left(1000 \mathrm{~W} / \mathrm{m}^{2}\right), T_{C}$ is cell temperature, $T_{C}{ }^{*}$ is cell temperature at standard test conditions $\left(25^{\circ} \mathrm{C}\right), P_{n G}$ is generator peak power at standard test conditions and $\gamma_{P}$ is temperature coefficient of maximum DC power.

Osterwald model has been used in combination with the nominal operation cell temperature model NOCT [32], presented in the following equation:

$T_{C}=T_{A}+(N O C T-20) \cdot G_{t} / 800$

where $T_{A}$ is ambient temperature and NOCT is nominal operation cell temperature. The model based on NOCT parameter has been selected because its performance is similar to more complex models for estimating cell temperature [33]. In addition, this parameter is available from the PV modules datasheets.

During this study, Osterwald model was found to be inaccurate for low levels of irradiance. In consequence, a model that defines PV module efficiency as a function of irradiance was included in the study. The expression of Eq. (16), first proposed by Beyer in [34], has been selected, where $e_{i}$ are fitting parameters that must be evaluated for every PV system. The effect of cell temperature is the same proposed by Osterwald.

$\eta=\eta\left(G_{t}, T_{C}\right)=\left[e_{1}+e_{2} \cdot G_{t}+e_{3} \cdot \log \left(G_{t}\right)\right] \cdot\left[1-\gamma \cdot\left(T_{C}-T_{C}{ }^{*}\right)\right]$

\section{Experimental validation}

\subsection{PV system description}

The methodology proposed in Section 2 has been validated using a BIPV system located at the Technical University of Madrid ("Lopez Araujo" building, ETSI Telecomunicacion). The modules are placed above the windows of the building south façade acting as sunshades; in winter, when the sun is low on the sky, the sunlight shines through the windows helping to warm up the building; in summer, with the sun higher in the sky, the PV generator blocks direct sunlight into the building reducing thermal load. Fig. 3 shows this system.

The system is formed by $282 \mathrm{PV}$ modules, oriented $8^{\circ}$ East and tilted $32^{\circ}$, distributed in 13 strings of serial connected modules. Nine of these strings are connected in parallel to a central inverter and the four remaining strings are connected to string inverters. 


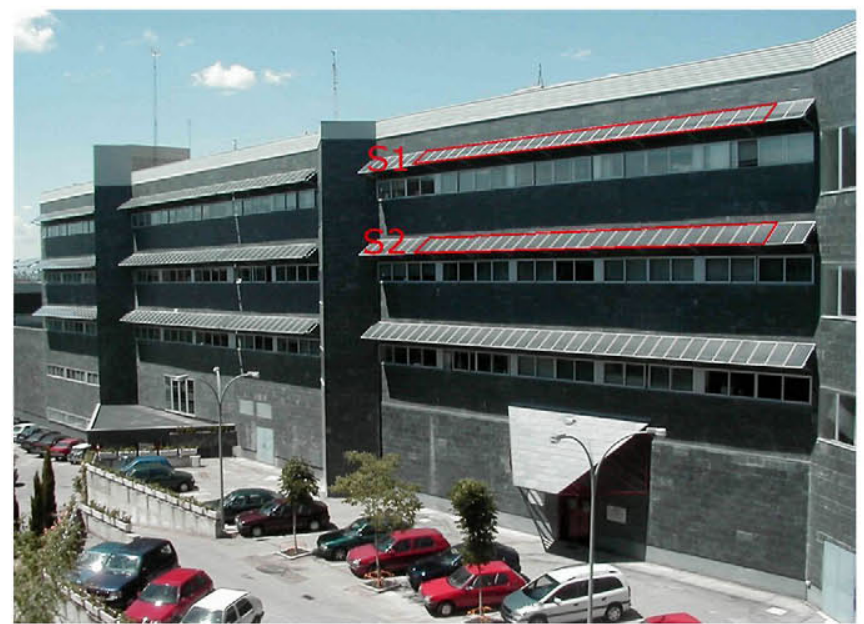

Fig. 3. PV system located on "Edificio López Araujo" building at ETSI. Telecomunicacion Madrid. (For interpretation of the references to colour in this figure caption, the reader is referred to the web version of this paper.)

The system also includes a meteorological station with the following sensors: two reference PV sensors of the same technology as the PV generator (crystalline silicon) for the measurement of irradiance $[35]^{1}$ and cell temperature [36] and a PT-100 resistor for the measurement of ambient temperature. Both PV sensors are of same technology as the modules, with same orientation and tilt angles as the modules and located on the roof top on a place free of shadows.

For this study information from two of the individual strings, S1 and S2, composed of 26 series connected PV modules each (outlined in red in Fig. 3), as well as data from the meteorological station, has been collected and analyzed. The reason for selecting these strings is that they are representative of BIPV systems where the PV generators act as sun shading elements, an architectural function that often implies partial shading in the PV modules. In fact, PV modules of string S2 are shaded during afternoon hours by the upper row S1. Thus, obstacle profiles for strings S1 and S2 are quite different, as shown in Fig. 4. These obstacles profiles have been traced from the most affected points of both PV generators: middle point of lower border for S1 and middle point of upper border for S2.

These two strings have been chosen because of their similarity: same number of PV modules and connection to the same inverter model. The PV generators' peak power at standard test conditions was characterized at the beginning of this study according to the procedure described by Caamaño et al. [37]. Since the strings are connected to the same inverter model, the MPPT strategy in both cases is the same. It is important to note that the MPPT efficiency of the inverters (and its associated error) is still present in the results presented in the next section. The conversion efficiency of both inverters was also characterized experimentally by measuring simultaneously inverters' input and output during a day with clear sky conditions, therefore, the characteristic parameters

\footnotetext{
${ }^{1}$ It must be noted that the international standard describing the requirements for the measurement of irradiance has changed since the deployment of this system and that the irradiance sensor does not follow the requirements of the new version of this standard. In particular, the voltage of the cell used as sensor, which is polarized by a shunt resistor that allows measuring the current, sometimes exceeds the limit of $3 \%$ of $V_{O C}$ as indicated by the new version of the standard. This leads to a slight underestimation at high values of incoming irradiance. However, this effect is small in crystalline silicon solar cells. This is corroborated by the fact that the errors of this methodology at high irradiance are lower than the errors at low irradiance (see Section 4).
}

obtained reflect inverters' real operation conditions (PV generator power voltage curve). For this experiment a precision wattmeter (model LMG 500 by ZIMMER Electronic Systems $\mathrm{GmbH}$ ) was used. The most relevant parameters of PV systems S1 and S2 are summarized in Table 1 . The values in table were obtained directly from the documentation provided by the manufacturers and the installer except those marked with a star $\left({ }^{*}\right)$ which were measured by the authors.

\subsection{Data set}

The monitoring system records every minute instantaneous values of the following parameters: in-plane irradiance, cell temperature, ambient temperature, $\mathrm{DC}$ power and $\mathrm{AC}$ power.

As mentioned before, the reference cell used to measure irradiance has the same orientation and slope as the PV generators. As the radiation model described in Section 3.1 calculates irradiation over a tilted surface from horizontal irradiation it is necessary to apply the criterion from Eq. (3) to extract values of horizontal irradiation from a typical meteorological year of Madrid [7]. Different values for the time interval $w$ have been tested: 0,1 , $5,10,15$ and 30 days.

The power on the AC and DC sides of the PV system has been measured by the built-in monitoring systems of the inverters. In order to determine its precision, measured power data were compared with measurements recorded by a precision wattmeter. For both inverters the mean absolute error and its associate standard deviation are given in Table 2.

The PV system under study has been monitored during one year: from March 2008 to February 2009. Before analysing model performance all records were checked in order to detect faulty data. Five days were excluded from the analysis because of partially or completely missing data. Additionally, 11 days were also excluded from the analysis in PV system S1 because the inverter monitoring system was not operating properly. Total number of excluded days amounts to less than $5 \%$ of the complete data set

\section{Results and discussion}

The methodology described in Section 2 has been used to estimate the electricity produced by the PV systems described in Section 3 . The instant values recorded by the monitoring system were processed in order to obtain average values every $10 \mathrm{~min}$. From these averages energy produced in spaces of $10 \mathrm{~min}$ has been calculated. The choice of a $10 \mathrm{~min}$ interval is a compromise between precision and interval size $[38,39]$.

Ten minute estimates and measured values were in turn averaged to get hourly values. A further filtering process was applied in order to remove values corresponding to in-plane irradiation lower than $5 \mathrm{Wh} / \mathrm{m}^{2}$. This filtering avoids the high uncertainty related to low values of power but it was kept as low as possible so that validation of the proposed methodology includes all weather conditions.

Comparison between the results of the proposed methodology and real values has been carried out at hourly, daily and yearly scales. The accuracy of daily and hourly electricity production estimates has been determined with the symmetric mean absolute percent error SMAPE [40]:

$\operatorname{SMAPE}(\%)=(1 / n) \cdot 100 \cdot \Sigma_{i}\left\|x_{i}-y_{i}\right\| /\left(x_{i}+y_{i}\right)$

where $n$ is the total number of points in the data set, days or hours; $x_{i}$ is the measured value and $y_{i}$ is the estimated value.

The SMAPE parameter overcomes the problem found at early morning or late afternoon, when irradiation values are low and a 


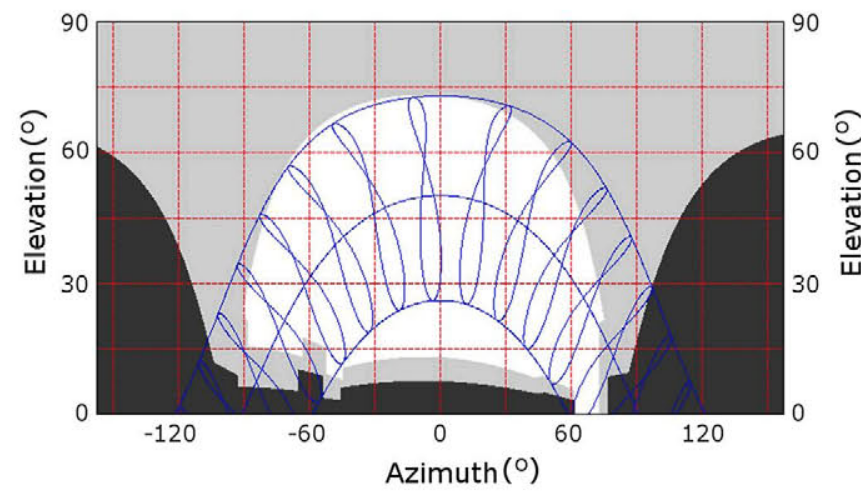

Fig. 4. Obstacle profiles for string $S 1$, in black, and string $S 2$, in light grey. Horizontal axis is azimuth and vertical axis is elevation.

Table 1

Relevant parameters for systems S1 and S2.

\begin{tabular}{lll}
\hline Parameter name & System 1 & System 2 \\
\hline Generator & & \\
Slope & $34^{\circ}$ & $34^{\circ}$ \\
Orientation & $-8^{\circ}$ & $-8^{\circ}$ \\
Peak power* & $2376 \mathrm{~W}$ & $2263 \mathrm{~W}$ \\
Modules in series & 26 & 26 \\
Modules in parallel & 1 & 1 \\
Module & & \\
NOCT & $47^{\circ} \mathrm{C}$ & $47{ }^{\circ} \mathrm{C}$ \\
$\beta$ & $-2.3 \mathrm{mV} /{ }^{\circ} \mathrm{C}$ & $-2.3 \mathrm{mV} /{ }^{\circ} \mathrm{C}$ \\
$\gamma$ & $0.004{ }^{\circ} \mathrm{C}$ & $0.004{ }^{-1} \mathrm{C}^{-1}$ \\
Cells in series & 33 & 33 \\
Cells in parallel & 2 & 2 \\
Inverter & & $2500 \mathrm{~W}$ \\
$P_{\text {max,I }}$ & $2500 \mathrm{~W}$ & $1.2 \%$ \\
$\mathrm{k}^{*}$ & $1.6 \%$ & $2.8 \%$ \\
$\mathrm{k} 1^{*}$ & $3.0 \%$ & $2.3 \%$ \\
$\mathrm{k} 2^{*}$ & $1.4 \%$ & \\
\hline
\end{tabular}

Table 2

Mean absolute errors and standard deviation of inverter built-in monitoring system.

\begin{tabular}{llc}
\hline & MAPE (\%) & $\sigma(\%)$ \\
\hline Inverter S1 & 1.5 & 3.4 \\
Inverter S2 & 2.3 & 3.5 \\
\hline
\end{tabular}

small error of few watts in electricity estimation is overweighted by a more traditional accuracy estimator like the mean absolute percentage error (MAPE). Another advantage of SMAPE is that it is less sensitive than MAPE to errors in measured values $\left(x_{i}\right)$. For annual estimates of electricity production, since they are single values, the percent error $\delta$ has been used:

$\delta=100 \cdot\left(y_{i}-x_{i}\right) / x_{i}$

Additionally, an external tool has been included in the study as a reference for a better assessment of the methodology performance: the commercial PVSyst software has been used. PVSyst has been chosen because it is a state of the art software widely used to analyze PV systems performance. It allows a thorough analysis of near shadings and PV strings can be split so that the behaviour of every module when shaded is modelled individually.

Tables 3 and 4 summarize the performance in the estimation of AC power by the models described in Section 2 and for all values of $w$ used to apply Eq. (3) as well as results provided by the reference
Table 3

Performance in the estimation of AC electricity for system S1 for different models and different window sizes.

\begin{tabular}{lllllll}
\hline $\begin{array}{l}\text { Model } \\
\left(\boldsymbol{E}_{\mathrm{AC}}\right)\end{array}$ & $\begin{array}{l}\text { window size } \\
(\mathbf{d a y s})\end{array}$ & $\begin{array}{l}\delta\left(\boldsymbol{E}_{\mathrm{AC}, \mathbf{a}}\right) \\
(\%)\end{array}$ & $\begin{array}{l}\text { SMAPEd } \\
(\%)\end{array}$ & $\begin{array}{l}\boldsymbol{\sigma d} \\
(\%)\end{array}$ & $\begin{array}{l}\text { SMAPEh } \\
(\%)\end{array}$ & $\begin{array}{l}\boldsymbol{\sigma} \boldsymbol{h} \\
(\%)\end{array}$ \\
\hline Osterwald & 0 & -6.1 & 8.4 & 9.7 & 23.6 & 33.4 \\
Osterwald & 1 & -4.1 & 6.4 & 8.1 & 21.4 & 32.5 \\
Osterwald & 5 & -3.9 & 6.5 & 8.5 & 20.8 & 32.7 \\
Osterwald & 10 & -4.3 & 7.0 & 8.6 & 21.1 & 33.1 \\
Osterwald & 15 & -4.3 & 6.9 & 7.9 & 20.8 & 32.6 \\
Osterwald & 30 & -4.4 & 7.3 & 9.9 & 21.3 & 32.9 \\
Beyer & 0 & -0.9 & 6.9 & 7.9 & 23.5 & 35.3 \\
Beyer & 1 & -0.8 & 5.1 & 6.6 & 20.8 & 33.5 \\
Beyer & 5 & -0.5 & 3.3 & 4.1 & 19.1 & 32.8 \\
Beyer & 10 & -0.6 & 3.0 & 3.4 & 18.9 & 32.7 \\
Beyer & 15 & -0.6 & 2.8 & 2.8 & 18.8 & 32.6 \\
Beyer & 30 & -0.6 & 3.1 & 3.7 & 18.8 & 32.3 \\
Reference & - & -11.3 & 11.7 & 2.7 & 54.7 & 61.7 \\
\hline
\end{tabular}

Table 4

Performance in the estimation of AC electricity for system S2 for different models and different window sizes.

\begin{tabular}{llrlrll}
\hline $\begin{array}{l}\text { Model } \\
\left(\boldsymbol{E}_{\mathrm{Ac})}\right.\end{array}$ & $\begin{array}{l}\text { window size } \\
(\text { days })\end{array}$ & $\begin{array}{l}\delta\left(\boldsymbol{E}_{\mathrm{AC}, \boldsymbol{a}}\right) \\
(\%)\end{array}$ & $\begin{array}{l}\text { SMAPEd } \\
(\%)\end{array}$ & $\begin{array}{l}\boldsymbol{\sigma d} \\
(\%)\end{array}$ & $\begin{array}{l}\text { SMAPEh } \\
(\%)\end{array}$ & $\begin{array}{l}\boldsymbol{\sigma} \boldsymbol{h} \\
(\%)\end{array}$ \\
\hline Osterwald & 0 & -6.2 & 15.4 & 18.7 & 48.4 & 52.7 \\
Osterwald & 1 & -3.1 & 11.2 & 14.7 & 44.0 & 51.6 \\
Osterwald & 5 & -2.5 & 7.6 & 9.0 & 40.8 & 50.9 \\
Osterwald & 10 & -3.3 & 6.7 & 7.8 & 40.6 & 51.2 \\
Osterwald & 15 & -3.3 & 6.3 & 6.7 & 39.9 & 51.1 \\
Osterwald & 30 & -3.6 & 6.6 & 9.3 & 39.6 & 51.2 \\
Beyer & 0 & 1.0 & 14.5 & 17.8 & 50.1 & 54.5 \\
Beyer & 1 & 1.6 & 10.5 & 15.7 & 47.4 & 55.8 \\
Beyer & 5 & 1.7 & 7.1 & 11.1 & 44.4 & 54.5 \\
Beyer & 10 & 1.5 & 6.4 & 10.0 & 44.5 & 54.8 \\
Beyer & 15 & 1.6 & 5.9 & 8.7 & 44.3 & 55.2 \\
Beyer & 30 & 1.5 & 5.7 & 7.2 & 43.7 & 54.8 \\
Reference & - & -14.1 & 15.1 & 5.9 & 69.4 & 67.8 \\
\hline
\end{tabular}

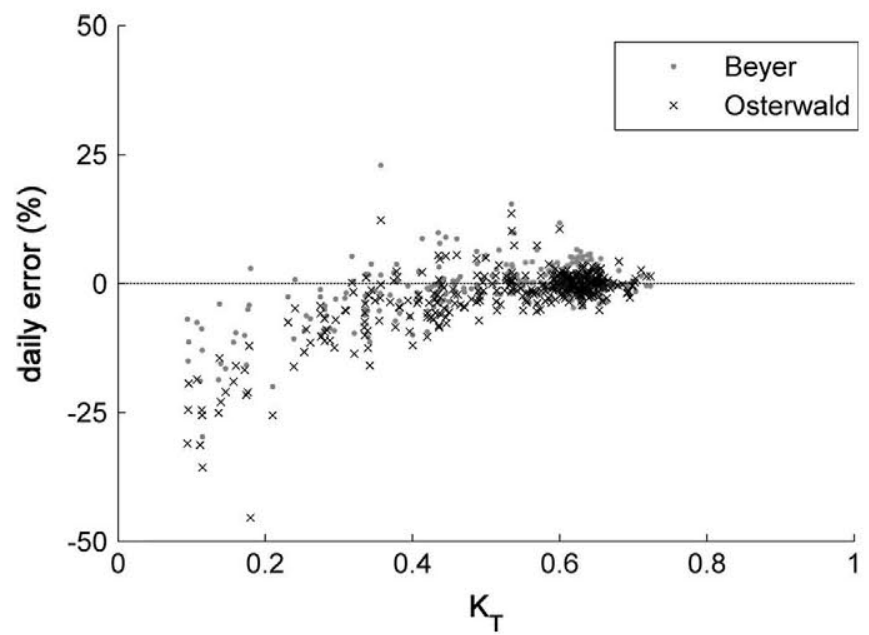

Fig. 5. Error in daily electricity estimations versus clearness index, comparison between Osterwald model and Beyer model for system S1.

model (PVSyst tool). Table 3 shows the results for system S1 and Table 4 for system S2. For daily and hourly values, along with the average error (SMAPE) its associated standard deviation, $\sigma$, is also given. Production estimates for system S1 are more accurate than 
Table 5

Average error (SMAPE) in daily electricity estimation for system S1.

\begin{tabular}{lcccc}
\hline Model & All (\%) & Sunny (\%) & Partially overcast (\%) & Fully overcast (\%) \\
\hline Osterwald & 6.5 & 2.8 & 5.4 & 19.0 \\
Beyer & 3.2 & 1.9 & 2.6 & 8.8 \\
Reference & 11.7 & 12.3 & 11.6 & 10.2 \\
\hline
\end{tabular}

Table 6

Average error (MAPE) in daily electricity estimation for system $\$ 2$.

\begin{tabular}{lrrrl}
\hline Model & All (\%) & Sunny (\%) & Partially overcast (\%) & Fully overcast (\%) \\
\hline Osterwald & 9.0 & 5.1 & 7.5 & 17.6 \\
Beyer & 7.1 & 4.5 & 7.6 & 16.6 \\
Reference & 15.1 & 15.8 & 15.2 & 13.5 \\
\hline
\end{tabular}

Table 7

Annual irradiation and daily mean irradiation for every day type.

\begin{tabular}{lllll}
\hline Irradiation & $\begin{array}{l}\text { All } \\
\left(\mathbf{k W h} / \mathbf{m}^{\mathbf{2}}\right)\end{array}$ & $\begin{array}{l}\text { Sunny } \\
\left(\mathbf{k W h} / \mathbf{m}^{\mathbf{2}}\right)\end{array}$ & $\begin{array}{l}\text { Partially overcast } \\
\left(\mathbf{k W h} / \mathbf{m}^{\mathbf{2}}\right)\end{array}$ & $\begin{array}{l}\text { Fully overcast } \\
\left(\mathbf{k W h} / \mathbf{m}^{\mathbf{2}}\right)\end{array}$ \\
\hline Total & 1641.5 & 814.2 & 732.6 & 82.4 \\
Daily (mean) & 4.5 & 6.1 & 4.5 & 1.3 \\
\hline
\end{tabular}

those for system S2 in all cases. This result is not unexpected because system S2 has a more severe obstacle profile than $\mathrm{S} 1$.

Comparison of results for different window sizes shows that a value of 0 for $w$, performs significantly worse than any other value for both systems; the remaining $w$ values exhibit a similar performance with absolute differences smaller than $2 \%$ within models. For annual estimations a value of 5 days for $w$ provides the best results in both systems. For daily and hourly values the lowest error corresponds to $w$ equal to 5,10 or 15 days, depending on model and system. The differences in performance for these three values are very close, with an absolute difference smaller than $0.5 \%$. However, the lowest standard deviation error is achieved with $w=15$ for all cases. These results show that windows corresponding to values of 0 and 1 for $w$ are small in size and provide poorly matching days. A bigger value for $w(5,10$ or 15$)$ performs better since, for each day, a wider collection of days exist from which to select the most adequate day. Further increasing the value of $w$ does not increase model performance anymore; a value of $w=30$ does not outperform smaller values, it only improves performance slightly for system S2.

Regarding comparison between models, the Beyer model provides the best results for system S1 while for system S2 Beyer model performs better for annual and daily values while Osterwald model performs better for hourly values. However, the differences between these two models are very narrow for both systems. When comparing with the reference model, both models tested in the study perform better. Interestingly, the reference model performs comparatively worse in the estimation of annual energy, the annual error being an order of magnitude larger. In addition, this model underestimates electricity production as annual error is negative for both systems. This fact is corroborated by the mean biased error (MBE), not shown on the tables, which is $-11.1 \%$ for system S1 and $-13.6 \%$ for S2.

Fig. 5 shows the errors in daily electricity estimation versus clearness index for system S1 and Beyer and Osterwald models. Daily errors are bigger for small values of clearness index for Osterwald model while for Beyer model daily error is uncorrelated with respect to clearness index. These results corroborate the appropriateness to include a model that incorporates the effect of irradiance level on PV module efficiency.

Finally, the performance of the proposed methodology has been compared with regard to meteorological conditions. The 365 days analyzed were divided into three categories:

- Clear days: the sky is free from clouds, diffuse fraction is below $10 \%$ and the irradiance profile for the whole day has a very characteristic bell-like shape.

- Partially casts days: the presence of scattered clouds in the sky covers and uncovers the sun repeatedly and irradiance changes its value very fast.

- Fully overcast days: the sky is totally covered by clouds; irradiance is irregular over the day but with low values, never exceeding $600 \mathrm{~W} / \mathrm{m}^{2}$.

Of the 365 analyzed days analyzed 136 were classified as sunny, 164 as partially overcast and 62 as fully overcast. Three days could not be classified because of missing data.

Average daily electricity estimation errors (SMAPE), separated into day types are shown in Tables 5 and 6 for Osterwald and Beyer models, using $w=5$ days as half window size. Average daily errors obtained from the reference model are also shown. The previous trends observed for all days also apply: electricity estimation is more accurate for system S1 than for S2 and the Beyer model performs better than Osterwald model. For these two models, performance for sunny and partially overcast days is significantly better than for fully overcast days, being performance on sunny days the best overall. The reference model, on the other hand, exhibits the opposite behaviour: it behaves better on fully overcast days than on sunny and partially overcast days. For fully overcast days, the reference model performs better than any other model for PV system S2 and it is comparable (slightly worse) to Beyer model for PV system S1. It must be noted that the impact of fully cast days in annual electricity production is significantly less than for clear and partially overcast days because of the differences in irradiation for every day type. In this sense, annual global irradiation and its breaking down into day typologies are presented in Table 7. Mean values of daily irradiation have been also included to enable comparisons between different weather conditions independent of the number of occurrences of every day during the year. Average daily irradiation for partially overcast days is $74 \%$ of irradiation available on sunny days while the percentage for fully overcast days is as low as $21 \%$. When looking at accumulated irradiation during the year, $50 \%$ of total annual irradiation corresponds to sunny days, $45 \%$ to partially overcast days and only $5 \%$ to fully overcast days while $17 \%$ of days, 62 out of 365 , were classified as fully overcast.

\section{Conclusions}

A new methodology for estimating the electricity produced by BIPV systems has been presented. This methodology incorporates a novel model for shading losses estimation developed specifically for BIPV systems. This model for shading losses estimation is based solely on geometrical considerations, an electrical model for the PV generator or information about modules internal structure and wiring are not needed. The methodology is validated with models to estimate the useful electricity a PV system can produce. Two alternative models to estimate electricity production have been tested. Noteworthy, the model that estimates electricity without a direct measurement of PV generator peak power, not usually performed on BIPV systems, provides results comparable, better in some cases, than the model requiring an explicit measurement of such characteristic. 
The methodology has been evaluated during one year with real data from two PV systems representative of BIPV. The evaluation has been performed over a wide range of irradiance values, starting from values as low as $5 \mathrm{~W} / \mathrm{m}^{2}$, covering all weather conditions. The error obtained in annual electricity estimation is below $1 \%$ for the best performing model. The SMAPE of daily electricity is below $3 \%$ for the same model. It has been also found that including the effect of reduced performance at low irradiance levels improves the estimation of the electricity yielded by a PV system. When compared to a reference model widely used in PV system performance analysis that can be considered representative of the actual state of the art, the methodology presented performed better than in annual, daily and hourly estimation of electricity production.

The proposed methodology is simple, easy-to-use and can provide fast, accurate results at low costs since it does not require specific equipment. These features make this technology suitable for application in the supervision of BIPV systems. Although the methodology has been designed specifically for building integrated PV systems it can be applied also to other types of PV systems different than building integrated.

\section{Acknowledgement}

Role of the funding source: This work has been financed by the Spanish Ministry of Education and Science (Plan Nacional I+D+I 204-2007) within the framework of the project Residential electricity demand side management with PV technology (ENE2007-66135).

\section{References}

[1] International Energy Agency. Trends in photovoltaic applications: survey report of selected IEA countries between 1992 and 2010. Report IEA-PVPS 2011; T1-20. Available for download at www.iea-pvps.org [last accessed 04/ $06 / 2012]$.

[2] RD/1699/2011 por el que se regula la conexión a red de instalaciones de producción de energía eléctrica de pequeña potencia, 2011. Spanish.

[3] Directive 2010/31/EU on the energy performance of buildings; 2010.

[4] Cereghetti N., Realini A., Benasconi A. Subsidy, check and monitoring of PV-plants: a happy ending story. In: Proceedings of the 20th European PV solar energy conference, Barcelona; 2005.

[5] Internacional Energy Agency. Cost and performance trends in grid-connected photovoltaic systems and case studies. Report IEA-PVPS; 2007. p. T2-06.

[6] Otani K, Kato K, Takashima T, Yamaguchi T, Sakuta K. Field experience with large-scale implementation of domestic PV systems and with large PV systems on buildings in Japan. Prog Photovolt: Res Appl 2004;12:449-59.

[7] Macagnan MH, Lorenzo E, Jimenez C. Solar radiation in Madrid. Int J Sol Energy 1994;16:1-14.

[8] Alonso-García MC, Ruiz JM, Herrmann W. Computer simulation of shading effects in photovoltaic arrays. Renew Energy 2006;31:1986-93.

[9] Alonso-Garcia MC, Ruiz JM, Chenlo F. Experimental study of mismatch and shading effects in the I-V characteristic of a photovoltaic module. Sol Energy Mater Sol Cells 2006;90:329-40.

[10] Di Piazza MC, Vitale G. Photovoltaic field emulation including dynamic and partial shadow conditions. Appl Energy 2010;87:814-23.

[11] Karatepe E, Boztepe M, Colak M. Development of a suitable model for characterizing photovoltaic arrays with shaded solar cells. Sol Energy 2007;81:977-92.

[12] Kawamura J, Naka K. Simulation of I-V characteristics of a PV module with shaded PV cells. Sol Energy Mater Sol Cells 2003;75:613-21.
[13] Rauschenbach HS. Electrical output of shadowed solar arrays. IEEE Trans Electron Devices 1971;18:483-90.

[14] Drif M, Pérez PJ, Aguilera J, Aguilar JD. A new estimation method of irradiance on a partially shaded PV generator in grid-connected photovoltaic systems. Renew Energy 2008;33:2048-56.

[15] Niewenda A, Heidt FD. SOMBRERO: a PC-tool to calculate shadows on arbitrarily oriented surfaces. Sol Energy 1996;58:253-63.

[16] Woyte A, Nijs J, Belmans R. Partial shadowing of photovoltaic arrays with different system configurations: literature review and field test results. Sol Energy 2003;74:217-33.

[17] Oozeki T, Izawa T, Otani K, Kurokawa K. An evaluation method of PV systems Sol Energy Mater Sol Cells 2003;74:687-95.

[18] Liu BYH, Jordan RC. The interrelationship and characteristic distribution of direct, diffuse and solar radiation. Sol Energy 1960;4:1-19.

[19] Perez R, Seals R, Ineichen P, Stewart T, Menicucci D. A new simplified version of the Perez diffuse irradiance model for tilted surfaces. Sol Energy 1987;39:221-31

[20] Hay JE, McKay DC. Estimating solar irradiance on inclined surfaces: a review and assessment of methodologies. Int I Sol Energy 1985;3:203-40.

[21] Reindl DT, Beckman WA, Duffie JA. Evaluation of hourly tilted surface radiation models. Sol Energy 1990;45:9-17.

[22] Feuermann D, Zemel A. Validation of models for global irradiance on inclined planes. Sol Energy 1992;48:59-66.

[23] Festa R, Ratto CF. Proposal of a numerical procedure to select reference years Sol Energy 1993;50:9-17.

[24] Petrakis M, Lykoudis S, Kassomenos P. A software tool for the creation of a typical meteorological year. Environ Softw 1996;11:221-7.

[25] Al-Rawi NA, Al-Kaisi MM, Asfer DJ. Reliability of photovoltaic modules Il. Interconnection and bypass diodes effects. Sol Energy Mater Sol Cells 1994:31:469-80.

[26] Silvestre S, Boronat A, Chouder A. Study of bypass diodes configuration on PV modules. Appl Energy 2009;86:1632-40.

[27] Órduz, R, Solórzano, J, Egido, MA, Román, E. Analytical study and evaluation results of power optimizers for distributed power conditioning in photovoltaic arrays. Prog Photovolt: Res Appl 2011; 10.1002/pip.1188. [published online].

[28] Martin N, Ruiz JM. Calculation of the PV modules angular losses under field conditions by means of an analytical model. Sol Energy Mater Sol Cells 2001;70:25-38.

[29] Schmidt, H, Sauer, DU. Praxisgerechte und praezise Modellierung von Wechselrichter-Wirkungsgraden. In: Proceddings of the IX International Sola Forum. Stuttgart, German; 1997.

[30] Osterwald CR. Translation of device performance measurements to reference conditions. Sol Cells 1986;18:269-79.

[31] Fuentes M, Nofuentes G, Aguilera J, Talavera DL, Castro M. Application and validation of algebraic methods to predict the behaviour of cristallyne silicon PV modules in Mediterranean climates. Sol Energy 2007;81:1936-48.

[32] International Electrotechnical Commision, Crystalline silicon terrestrial photovoltaic (PV) modules. Design qualification and type approval; 2005. IEC 61215 ed2.0, 2005

[33] Masa-Bote D., Caamaño E., Improved methodology for system performance analysis. In: Proceedings of the 24th European PV solar energy conference. Hamburg; 2009.

[34] Beyer H.G., Betcke J., Drews A., Heinemann D., Lorenz E., Heilscher G., et al Identification of a general model for the MPP performance of PV-modules for the application in a procedure for the performance check of grid connected systems. In: Proceedings of the 19th European PV solar energy conference, Paris; 2004.

[35] International Electrotechnical Commision. Photovoltaic devices. Part 2: Requeriments for reference solar cells. IEC 60904-2; 1994

[36] International Electrotechnical Commision. Photovoltaic devices. Part 5: deter mination of the equivalent cell temperature (ECT) of photovoltaic (PV) devices by the open-circuit voltage method. IEC 60904-5 ed2.0; 2011.

[37] Caamaño E, Lorenzo E, Zilles R. Quality control of wide collection of PV Modules: lessons learned from the IES experience. Prog Photovolt: Res Appl 1999;7:137-49.

[38] Ransome S., Funtan P. Why hourly averaged measurement data is insufficient to model PV system performance accurately. In: Proceedings of the 20th European PV solar energy conference. Barcelona; 2005.

[39] Perpiñan O, Lorenzo E, Castro A, Eyras R. On the complexity of radiation models for PV energy production calculation. Sol Energy 2008;82:125-31.

[40] Armstrong JS. Long range forecasting. 2nd ed.. New York: Wiley; 1985 